\title{
Charcot neuroarthropathy of the hand in a diabetic patient after upper extremity trauma
}

\author{
Fazıl Kulakıı1, Canan Çulha'², Seval Akdemir ${ }^{2}$ \\ ${ }^{I}$ Department of Physical Medicine and Rehabilitation, Giresun University School of Medicine, Giresun Ilhan Özdemir Training \\ and Research Hospital, Giresun, Turkey \\ ${ }^{2}$ Department of Physical Medicine and Rehabilitation, Ankara Physical Medicine and Rehabilitation Training and Research Hos- \\ pital, Ankara, Turkey
}

\begin{abstract}
Charcot neuroarthropathy is a progressive degenerative arthritis which affects nearly every joint of the body associated with an underlying central or peripheral neurological disorder and can cause severe disability. The joints most frequently involved are lower limbs especially foot, shoulder and elbow. However, Charcot neuroarthropathy of the hand is quite rare. Herein, we present a 50-year-old diabetic female patient with CNA of the right hand after upper extremity trauma. Major or minor traumas in diabetic patients can increase the risk of CNA on upper extremity as a complication of diabetes mellitus.
\end{abstract}

Eur Res J 2018;4(3):258-261

Keywords: Charcot neuroarthropathy, diabetes mellitus, trauma

\section{Introduction}

Charcot neuroarthropathy (CNA) firstly recognized in 1868 by Jean Marie Charcot, still remains a clinical problem. There is a progressive disorganization of architecture in the intensive joint, leading to painless joint swellings with radiological evidence of pronounced bone destruction as well as new bone formation in abnormal sites [1]. Due to their role in weight-bearing activities, the joints of the lower extremity are most commonly involved in this pathology. CNA of the upper extremity occurs in shoulder most frequently [2]. However, CNA of the hand is quite uncommon. In this study, we aimed to present a diabetic patient with CNA of the right hand after upper extremity trauma.

\section{Case Presentation}

A 50-year-old female patient admitted to our hospital with painless joint swelling, movement limitation and deformity complaints on the right hand. Two years ago, she dropped on her right arm and after this trauma, swelling, pain and weakness complaints were developed on her arm. She appealed to a hospital with these complaints and was diagnosed with shoulder dislocation, humeral fracture and fracturedislocation of the wrist. After plaster cast treatment for 6 weeks, swelling, pain, weakness and movement limitation complaints were in progress and she was admitted to a centre for carrying out physical therapy and rehabilitation program. After physical therapy and rehabilitation program, pain and swelling complaints 
decreased but movement limitation and weakness were in progress. Although movement limitation decreased slightly, she could do her daily works as a farmer by using her right hand predominantly but for skilled movements, she used her left hand. She appealed to our hospital with complaint of movement limitation, painless swelling and deformity on the right hand. The patient had newly diagnosed diabetes mellitus, hypertension, hyperlipidemia, hepatitis-B and chronic bronchitis as systemic diseases. She did not have a knowledge about individuals with these symptoms in her family. No abnormality was detected on rheumatological questioning. There was no pathologic finding on physical examination of systems except locomotor system. Left upper extremity and bilateral lower extremity findings were normal. Although right shoulder flexion was limited at 145 degrees actively and passively, other ranges of motion (ROM) of shoulder were normal. Elbow flexion and extension ranges were normal, but supination and pronation were limited at last 10 degrees actively and passively. Wrist ROM was normal. First metacarpophalangeal joint (MCP) and interphalangeal joint ranges were normal. MCP flexion of 2-3-4 fingers were limited at 40 degrees. Flexion of 2-3-4-5 proximal interphalangeal (PIP) were limited at 80 degrees but extension was normal. Extension of 2-34-5 distal interphalangeal (DIP) were limited at last 5 degrees. Flexion of 2-3-4-5 DIP were complete but all the fingers were standing position of flexion (Figure $1)$.

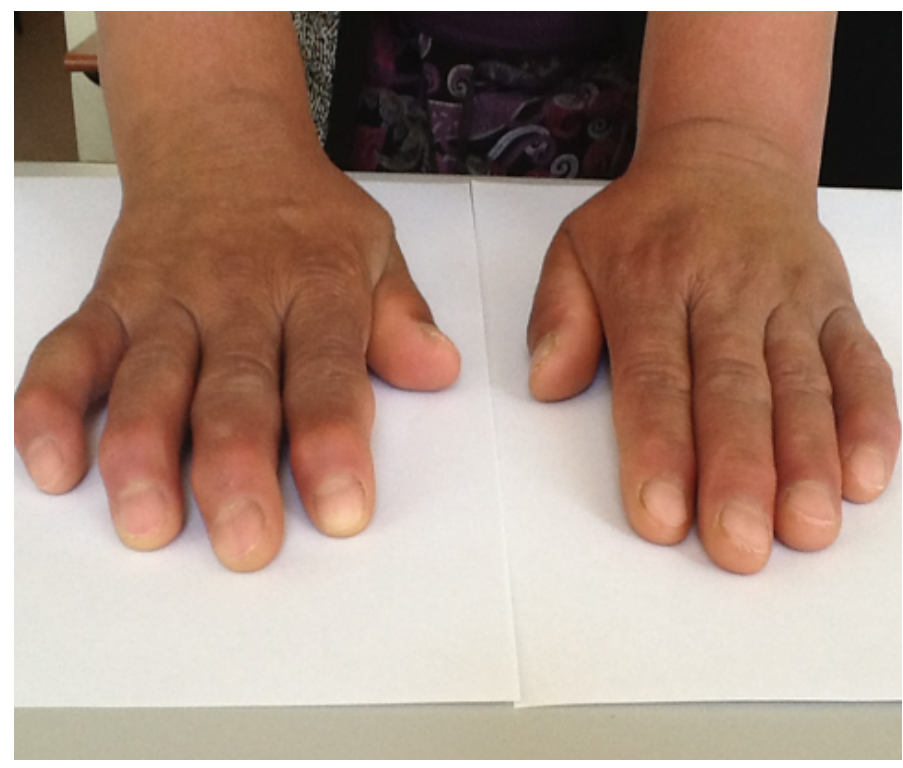

Figure 1. Painless swellings and deformities on the right proximal interphalangeal and distal interphalangeal joints
Although upper extremity motor examination was normal (5/5) around the elbow, wrist and fingers in present ROM, muscle strength of right shoulder abduction and flexion were $4 / 5$. A diffuse hypoesthesia and hypoalgesia was detected on right upper extremity, deep tendon reflexes were normal, and no pathologic reflexes were found. Tinnel's and Phalen's sign tests of right wrist were positive. Hypertrophic, erosive and degenerative changes were detected on DIP and PIP joints of right hand on $\mathrm{x}$-ray but there were no changes on left hand (Figure 2).

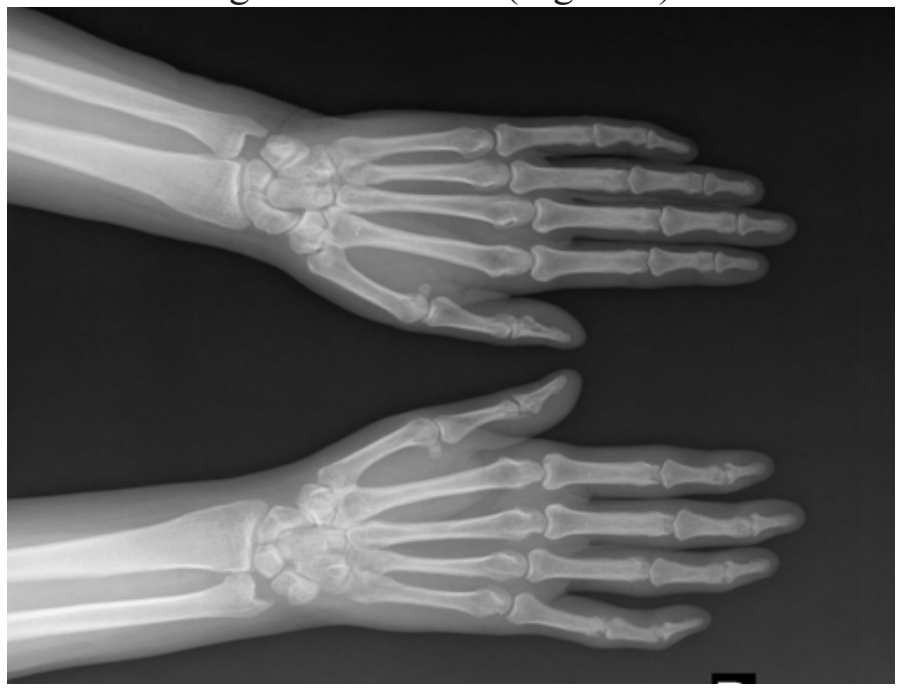

Figure 2. Osteophytes, hypertrophic, erosive and destructive changes on proximal interphalangeal and distal interphalangeal joints of right hand, no degenerative changes on left hand.

There were no considerable changes on the x-rays of shoulder, arm, elbow, forearm and wrist. The results of the patient's blood analysis were associated with diabetes mellitus, hyperlipidemia and hepatitis-B. In addition, serum erythrocyte sedimentation rate and Creactive protein values were normal. Rheumatoid factor, uric acid, anti-nuclear antibody, extractable nuclear antigens values were normal. Free T3, Free $\mathrm{T} 4$, thyroid stimulating hormone, vitamin B12, folate and parathyroid hormone values were normal but 25 hydroxyvitamin $\mathrm{D}$ was detected $11.8 \mathrm{ng} / \mathrm{mL}$ described as lower. Right mild pan-brachial plexopathy and moderate carpal tunnel syndrome (CTS) was detected on electroneuromyography (ENMG). After these evaluations, the patient was diagnosed as CNA caused by diabetes mellitus, brachial plexopathy and CTS and the treatment was planned in this direction. 


\section{Discussion}

CNA is a limb-threatening, destructive process that occurs in patients with neuropathy associated with central and peripheral neurological diseases such as diabetes mellitus, syphilis, syringomyelia and peripheral nerve injuries. Diabetes mellitus is the leading cause of CNA and current estimates of its prevalence range from $0.08 \%$ in the general diabetic population to $13 \%$ in high-risk diabetic patients [3]. In addition, syringomyelia is the leading cause of CNA of the upper extremity, with the shoulder and elbow being the most commonly affected joints [2]. CNA is one of the most destructive complications of diabetes mellitus, leading to subluxation, dislocation, deformity, and ulceration of the foot and other joints. The basic factor in pathogenesis of CNA is lack of sensorial stimulus coming from joint. Deficiency in the sensations of pain and proprioception causes prolonged trauma to joint. Reiterative traumas to joint triggers fibrillation and fragmentation of cartilage and this situation causes formation of loose bodies in the joint space [4]. Despite severe clinical findings, pain is not a disturbing symptom for patients and this is the most important factor for differential diagnosis. However, osteoarthritis, monoarticular and poliarticular diseases, infective artrhopathy, crystal deposition diseases, trauma, hemarthrosis, intermittent arthritis and osteonecrosis should be considered in the differential diagnosis [5]. In our patient, the most distinctive feature is severe joint destruction in DIP and PIP joints of right hand without pain. Our patient had a mild brachial panplexopathy and moderate CTS on the right side, but we evaluated the patient two years after the injury. The patient said that there was no muscle strength on her right arm after injury for a few months. After this duration the muscle strength increased slowly and movement limitations occured in first 6 months after injury on the paretic side, so we thought that there was a severe brachial plexus injury on the right side after injury but a good healing occured on the brachial plexus and muscle strength increased little by little. The patient was diagnosed as CNA because the patient had painless destructive arthropathy on DIP and PIP joints and newly diagnosed and long time untreated diabetes mellitus as a major cause of CNA. In addition, the patient was diagnosed brachial plexopathy and CTS by using ENMG are other causes of CNA. Furthermore, we found abnormal neurological findings on the right upper extremity concomitant to destructive arthropathy. The rheumatological questioning and acute phase reactants were normal and severe destructive arthropathy was detected on radiological images. Interestingly, destructive changes were detected on DIP and PIP joints but there was no change on wrist joint. According to above symptoms and findings, other diseases were excluded, and the patient was diagnosed with CNA associated with diabetes mellitus and peripheral nerve injury. In patients with diabetes mellitus, CNA mainly affects major weight-bearing joints, especially the foot and ankle. CNA rarely affects joints other than the foot and ankle in diabetes mellitus patients and only a very few cases of CNA of the wrist have been reported so far $[6,7]$. The explanation of this rarity is the lesser degree of upper extremity involvement due to sensorimotor and autonomic neuropathy in comparison with the lower limb. In addition, CNA affects weight-bearing joints primarily, like the foot and ankle, which are exposed to continuous trauma [6]. The patient used her right hand dominantly and her occupation (farmer) was a risk factor for developing CNA because of constituting micro and macro trauma to the hand. We think that, newly diagnosed and uncontrolled diabetes mellitus and presented nerve injuries caused to reduction of pain and proprioceptive sensation so the trauma did not disturb the patient. Traumatic phase continued and a trauma-inflammation circle occured, so this situation could accelerate the development of CNA. There is yet no specific pharmacological treatment for CNA. Some clinicians also prescribe bisphosphonates in the early stages of treatment, as the bone mineral density of the affected foot is low [8]. Off-loading the affected joint with bracing for at least 2-3 months seems to be essential. Aside from pain management, good glycemic control is also a crucial part of the therapy [9]. Keep in mind that a patient with neuropathy may be unaware of injury to the joint, so patient and family education is so important. Surgery is reserved for severe ankle and midfoot deformities that are susceptible to skin ulcerations and that make braces and orthotic devices difficult to use [10].

\section{Conclusion}

Our patient had uncontrolled diabetes mellitus. In addition, a major trauma occurred to the right upper extremity and symptoms and findings were coming out after this trauma so we must keep in mind that major or minor traumas in diabetic patients can 
increase the risk of CNA on upper extremity as a complication of diabetes mellitus.

\section{Informed consent}

Written informed consent was obtained from the patient for the publication of this case report.

\section{Conflict of interest}

The authors declared that there are no potential conflicts of interest with respect to the research, authorship, and/or publication of this article.

\section{References}

[1] Ekim A, Armağan O. Neuropathic arthropathy caused by syringomyelia in different joints and lesion of brachial plexus at right upper extremity: a case report. Ağr1 2007;19:54-8.
[2] Snoddy MC, Lee DH, Kuhn JE. Charcot shoulder and elbow: a review of the literature and update on treatment. J Shoulder Elbow Surg 2017;26:544-52.

[3] Frykberg RG, Belczyk R. Epidemiology of the Charcot foot. Clin Podiatr Med Surg 2008;25:17-28.

[4] Elman MH. Neuropathic joint disease (Charcot joints). In: McCarty DJ, editor. Arthritis and allied conditions. 11th ed. Philadelphia: Lea and Febiger; 1989;1255-72.

[5] Jones EA, Manaster BJ, May DA, Disler DG. Neuropathic osteoarthropathy: diagnosis and differential diagnosis. Radiographics 2000;20:S279-93.

[6] Lambert AP, Close CF. Charcot neuroarthropathy of the wrist in type 1 diabetes. Diabetes Care 2005;28:984-5.

[7] Bayne O, Lu EJ. Diabetic Charcot's arthropathy of the wrist.Case report and literature review. Clin Orthop Relat Res 1998;357:122-6.

[8] Young MJ, Marshall A, Adams JE, Selby PL, Boulton AJ. Osteopenia, neurological dysfunction, and the development of Charcot neuroarthropathy. Diabetes Care 1995;18:34-8.

[9] Çağlayan G, Kılıç E, Özçakar L. Acute charcot arthropathy of the wrist in a diabetic patient. Rheumatol Int 2013;33:2959-60.

[10] Botek G, Anderson MA, Taylor R. Charcot neuroarthropathy: an often overlooked complication of diabetes. Cleve Clin J Med 2010;77:593-9. 